\title{
EVALUATION OF INNO-LIPA MYCOBACTERIA V2 ASSAY FOR IDENTIFICATION OF RAPIDLY GROWING MYCOBACTERIA
}

\author{
Lidia García-Agudo $^{1 *}$, Iría Jesús ${ }^{1}$, Manuel Rodríguez-Iglesias ${ }^{1}$, Pedro García-Martos ${ }^{2}$ \\ ${ }^{1}$ Microbiology Service. Hospital de Puerto Real, Carretera Nacional IV km 665, 11510 Puerto Real, Cádiz, Spain; ${ }^{2}$ Microbiology \\ Service. Hospital Puerta del Mar, Ana de Viya 21, 11009 Cádiz, Spain.
}

Submitted: August 25, 2010; Returned to authors for corrections: October 25, 2010;Approved: May 16, 2011.

\begin{abstract}
A total of 54 rapidly growing mycobacteria (RGM) isolated from patients attended in the two hospitals of Cádiz Bay (Spain) were selected during a seven-year-period (2000-2006) in order to evaluate the INNOLiPA Mycobacteria v2 assay for mycobacterial identification, based on the reverse hybridization principle. The strains were cultured in Löwenstein-Jensen and Middlebrook 7H9 media and identified to the species level by sequencing of the 16S rRNA, PCR-restriction enzyme analysis of the $h s p 65$ gene, conventional tests and INNO-LiPA Mycobacteria v2 assay. By the molecular methods we identified a total of 12 different species: 23 Mycobacterium fortuitum, 11 M. chelonae, 10 M. abscessus, 2 M. senegalense, 1 M. alvei, 1 M. brumae, 1 M. mageritense, 1 M. mucogenicum, 1 M. neoaurum, 1 M. peregrinum, $1 \mathrm{M}$. septicum and $1 \mathrm{M}$. smegmatis. Fifty two strains $(96.3 \%)$ were correctly identified by conventional techniques and 47 strains $(87.0 \%)$ by INNO-LiPA Mycobacteria v2 assay. We find INNO-LiPA Mycobacteria v2 assay simple to perform but it provides few advantages in comparison with conventional methods and sometimes needs complementary tests to identify Mycobacterium fortuitum complex, M. chelonae complex and specific species due to the great heterogeneity in the RGM group.
\end{abstract}

Key words: Mycobacteria; rapidly growing mycobacteria; M. fortuitum; M. chelonae; M. abscessus.

\section{INTRODUCTION}

Rapidly growing mycobacteria (RGM) are ubiquitous in nature and widespread in water, soil, poultry and animals. Interest in RGM is rising in the last three decades due to a notorious increase of post-traumatic and post-surgical infections, localized and disseminated, and outbreaks of infection by contaminated medical equipment. RGM have been described in pulmonary infections, keratitis, endophthalmitis, suppurative arthritis, osteomyelitis, endocarditis, meningitis, peritonitis, chronic urinary tract infection, otitis media related to tympanostomy tube insertion and catheter-associated bacteremia. The members of the Mycobacterium fortuitum complex, $M$. chelonae and $M$. abscessus are the species most frequently associated with human infections; the rest of species are minority and occasionally reported $(2,3,6,13,14)$.

*Corresponding Author. Mailing address: Unidad de Microbiología, Hospital de Tomelloso, Vereda de Socuéllamos s/n, 13700 Tomelloso (Ciudad Real, Spain).; Tel: +34 926525837.; E-mail: lidiagarciaagudo@gmail.com 
Identification of new species of RGM producing nosocomial infections and serious infections in oncological and immunocompromised patients has been possible thanks to the development of modern microbiological diagnosis systems and genetic methods, such as the sequencing of bacterial $16 \mathrm{~S}$ rRNA region or the restriction enzyme pattern analysis of the hsp65 gene (PRA), which encodes for the $65-\mathrm{kDa}$ heat shock protein $(1,8,10)$. In spite of several target genes have been used for the mycobacterial identification, the most common is the 16S rRNA, considered the gold standard as it flanks species-specific sequences (11). The 65-kDa heat shock protein contains unique epitopes as well as common epitopes to various species of mycobacteria. Beyond other PCR-based and hybridization methods for differentiating mycobacterial species limitation, PRA does not require hybridization to a panel of species-specific probes. Those and other molecular techniques have already been proved to be very useful to identify $M$. tuberculosis complex $(12,13)$.

INNO-LiPA Mycobacteria v2, designed to amplify the mycobacterial 16S-23S rRNA internal transcribed spacer region (ITS), which is more discriminative than the 16S rRNA region itself, is a new genetic test for the simultaneous identification of 16 different species within the genus Mycobacterium on broth or solid cultures (4, 5, 7, 9, 14, 15).

Our purpose was to assess the diagnostic value of this new molecular method for the identification of RGM to the species level from positive broth cultures.

\section{MATERIALS AND METHODS}

During a seven-year-period (2000-2006), we studied 54 strains of RGM isolated from patients attended in Puerto Real University Hospital and Puerta del Mar University Hospital (Cádiz, Spain). All specimens were cultured on LöwensteinJensen and Middlebrook 7H9 media (Becton-Dickinson, UK). Non-sterile specimens were previously submitted to digestion and decontamination with $\mathrm{N}$-acetyl-cysteine and sodium hydroxide to eliminate the contaminating flora.

\section{Sequencing of the 16S rRNA gene}

PCR of the sequence coding for 16S rRNA was performed with the primers PMyc14bio [5'-GRGRTACTCGAGTGGCGA AC] and PMyc7 [5'-GGCCGGCTACCCGTCGTC] (11). The presence of amplified DNA was visualized by agarose gel electrophoresis and staining with ethidium bromide. Direct sequencing of the PCR product was determined on the automated ABI PRISM 310 sequencer (Applied Biosystems, USA), according to the manufacturer's instructions. The identification of the strain was completed by comparison of the nucleotide sequence with a library of known sequences. The databases for this purpose, which are available in the internet, are the GenBank, the DNA Data Bank of Japan (DDBJ), that of the European Molecular Biology Laboratory (EMBL) and the Ribosomal Differentiation of Medical Microsystems database (RIDOM). In our study, the nucleotide sequences were aligned with those available in the GenBank database, provided by the National Center for Biotechnology Information.

\section{PCR-restriction enzyme analysis}

PCR-restriction enzyme analysis included a previous PCR of a segment of the $65-\mathrm{kDa}$ heat shock protein gene (hsp65), amplified by two specific primers, Tb11 [5'-ACCAACGATGG TGTGTCCAT] and Tb12 [5'-CTTGTCGAACCGCATACC CT] (16). The presence of amplified products was confirmed by agarose gel electrophoresis. Restriction analysis was performed by BstEII and HaeIII enzymes. After digestion fragments were visualized by ethidium bromide staining and UV light onto an agarose gel. Restriction patterns were analyzed with Quantity One computer program (BioRad, USA) and aligned with the available patterns in the database of this program.

Sequencing of the 16S rRNA and PCR-restriction enzyme analysis of the hsp65 gene were carried out in the Centro Nacional de Microbiología (Instituto de Salud Carlos III; Madrid, Spain).

\section{Conventional methods}

Conventional methods included phenotypical techniques 
(growth rate and growth temperature, pigmentation, colonial morphology) and biochemical tests (nitrate reduction, arylsulfatase and urease production, tolerance to $5 \% \mathrm{NaCl}$, carbohydrate utilization (mannitol, inositol, sorbitol), tween 80 hydrolysis, growth on MacConkey agar without crystal violet).

\section{INNO-LiPA Mycobacteria v2 assay}

Identification by INNO-LiPA Mycobacteria v2 assay (Innogenetics, Belgium) required a previous mycobacterial cell lysis for releasing nucleic acids. From broth cultures, $2 \mathrm{ml}$ were centrifuged at $13,000 \mathrm{rpm}$ for 15 minutes, the pellet was resuspended in TE buffer $(10 \mathrm{mM}$ Tris $\mathrm{HCl}, 1 \mathrm{mM}$ EDTA [pH 8]) and heat inactivated at $95^{\circ} \mathrm{C}$ for 30 minutes, followed by further centrifugation at 13,000 rpm for 10 seconds, freezing at $220^{\circ} \mathrm{C}$ for 30 minutes, vortexing, and final centrifugation as above. Ten microliters of supernatant was used for amplification in the presence of biotinylated primers complementary to the sequences flanking the $16 \mathrm{~S}-23 \mathrm{~S}$ ribosomal RNA spacer region, which had the sequences TTGTACACACCGCCCGTCA and CGATGCCAAGGCATC CACC (17). Amplification was carried out in an automated thermocycler (GenAmp PCR System 2700; Applied Biosystems, USA) following the profile indicated by the manufacturer. The success of the amplification step was checked by gel electrophoresis. The amplified product was then hybridized with specific oligonucleotide probes immobilized as parallel lines on membrane-based strips, bound with streptavidin labelled with alkaline phosphatase and incubated with 5-bromo-4-chloro-3-indolyl phosphate and nitroblue tetrazolium chromogen, resulting in a purple-brown precipitate if hybridization had ocurred. Hybridization and detection of the amplified product were performed in the automated instrument Auto-LiPA (Innogenetics, Belgium). Appearance of a clearly visible line was considered as a positive result. The manufacturer provides an interpreting diagram which includes a MFO line for the M. fortuitumperegrinum complex, a MSM line for M. smegmatis and three more for the $M$. chelonae complex: MCH-1 (groups I, II, III, IV and M. abscessus), MCH-2 (group III and M. abscessus) and MCH-3 (group I). This procedure has been previously described $(7,14)$.

\section{RESULTS}

We isolated a total of 54 strains of RGM (9.4\%) from 576 specimens received in our laboratories during the study period. The distribution of specimens were: 34 respiratory secretions, 9 cutaneous abscesses, 4 urines, 4 corneal ulcers, 1 lymphatic nodule, 1 peritoneal liquid and 1 blood. Strains were identified by molecular methods as belonging to 12 different species: 23 M. fortuitum, 11 M. chelonae, 10 M. abscessus, 2 M. senegalense, 1 M. alvei, 1 M. brumae, 1 M. mageritense, $1 \mathrm{M}$. mucogenicum, $1 \mathrm{M}$. neoaurum, $1 \mathrm{M}$. peregrinum, $1 \mathrm{M}$. septicum and 1 M. smegmatis (Table 1).

According to the phenotypic characteristics, 53 strains (98.1\%) were recognized as nonchromogen, though one presented yellowish tonalities ( $M$. brumae) and another produced a late orange pigment (M. smegmatis). Only one strain was considered scotochromogen for producing an orange pigment (M. neoaurum).

Fifty-two out of 54 strains $(96.3 \%)$ were correctly identified by conventional methods. Biochemical tests results are shown in Table 2. The two misidentified strains through their biochemical profile were $M$. senegalense, which did not grow in mannitol and was identified as $M$. mageritense, and $M$. alvei, identified as $M$. fortuitum. INNO-LiPA Mycobacteria v2 assay accurately identified 47 strains (87.0\%). The seven misidentified or unidentified strains were: one strain of $M$. chelonae identified within the $M$. fortuitum-peregrinum complex; one strain of $M$. mageritense identified within the MCH-1 complex (I, II, III, IV, M. abscessus); one of $M$. senegalense, one of $M$. mucogenicum and one of M. abscessus, which gave no result; and two strains corresponding to $M$. brumae and M. neoaurum, whose identification is not included in the technique. Discarding these last two species, the 
accuracy for the identification of the rest of species was of 90.4\%. Otherwise, two strains of $M$. chelonae were identified within the MCH-2 complex (III, M. abscessus) and five of $M$. abscessus within the MCH-1 complex (I, II, III, IV, $M$. abscessus). Outcomes by phenotypical tests and by INNOLiPA Mycobacteria v2 assay are shown in Table 3.

Table 1. Sources of isolation of the 54 rapidly growing mycobacteria strains.

\begin{tabular}{lcccccccccccc}
\hline Sources & MFO $^{\mathbf{a}}$ & MCH & MAB & MSE & MAL & MBR & MMA & MMU & MNE & MPE & MSP & MSM \\
\hline Respiratory secretions & 15 & 6 & 5 & 2 & 1 & 1 & 1 & 1 & 0 & 1 & 1 & 0 \\
Cutaneous abscesses & 5 & 2 & 1 & 0 & 0 & 0 & 0 & 0 & 0 & 0 & 0 & 1 \\
Urines & 3 & 0 & 1 & 0 & 0 & 0 & 0 & 0 & 0 & 0 & 0 & 0 \\
Lymphatic nodules & 0 & 0 & 1 & 0 & 0 & 0 & 0 & 0 & 0 & 0 & 0 & 0 \\
Peritoneal liquids & 0 & 1 & 0 & 0 & 0 & 0 & 0 & 0 & 0 & 0 & 0 & 0 \\
Corneal ulcers & 0 & 2 & 2 & 0 & 0 & 0 & 0 & 0 & 0 & 0 & 0 & 0 \\
Blood & 0 & 0 & 0 & 0 & 0 & 0 & 0 & 0 & 1 & 0 & 0 & 0 \\
TOTAL & 23 & 11 & 10 & 1 & 1 & 1 & 1 & 1 & 1 & 2
\end{tabular}

${ }^{\mathrm{a}} \mathrm{MFO}=$ M. fortuitum, $\mathrm{MCH}=M$. chelonae, $\mathrm{MAB}=M$. abscessus, $\mathrm{MSE}=M$. senegalense, $\mathrm{MAL}=$ M. alvei, $\mathrm{MBR}=M$. brumae, $\mathrm{MMA}=$ M. mageritense, $\mathrm{MMU}$ $=M$. mucogenicum, $\mathrm{MNE}=M$. neoaurum, $\mathrm{MPE}=M$. peregrinum, $\mathrm{MSP}=M$. septicum, $\mathrm{MSM}=M$. smegmatis

Table 2. Biochemical profile of the 54 rapidly growing mycobacteria strains.

\begin{tabular}{|c|c|c|c|c|c|c|c|c|c|c|c|}
\hline Species & Strains & $42^{\circ} \mathrm{C}$ & $\mathbf{N I T}^{\mathrm{a}}$ & ARS & URE & TW80 & $\mathrm{NaCl}$ & MAC & MAN & INO & SOR \\
\hline M. abscessus & 10 & - & - & + & + & - & + & + & - & - & - \\
\hline M. alvei & 1 & - & + & + & + & + & - & - & - & - & - \\
\hline M. brumae & 1 & - & + & - & + & + & - & - & - & + & - \\
\hline M. chelonae & 11 & - & - & + & + & $v^{c}$ & - & - & - & - & - \\
\hline M. fortuitum & 23 & $v^{b}$ & + & + & + & $v^{d}$ & + & - & - & - & - \\
\hline M. mageritense & 1 & + & + & + & + & - & + & + & - & - & - \\
\hline M. mисоgenicum & 1 & - & - & + & + & + & - & - & + & - & - \\
\hline M. neoaurum & 1 & - & + & - & + & + & - & - & + & + & - \\
\hline M. peregrinum & 1 & - & + & + & + & - & + & + & + & - & - \\
\hline M. senegalense & 2 & + & + & + & + & - & + & - & + & - & - \\
\hline M. septicum & 1 & + & + & + & + & - & + & + & + & - & - \\
\hline M. smegmatis & 1 & - & + & - & + & + & + & + & + & + & - \\
\hline
\end{tabular}

${ }^{\mathrm{a}} \mathrm{NIT}=$ nitrate reduction; $\mathrm{ARS}=$ arylsulfatase production; $\mathrm{URE}=$ urease production: $\mathrm{TW} 80=$ tween $80 \mathrm{hydrolysis} ; \mathrm{NaCl}=$ tolerance to $5 \% \mathrm{NaCl} ; \mathrm{MAC}=$ growth on MacConkey agar without crystal violet; MAN = mannitol utilization; INO = inositol utilization; SOR = sorbitol utilization

${ }^{\mathrm{b}}$ Positive in 14 strains $(61 \%) ;{ }^{\mathrm{c}}$ Positive in 5 strains $(45 \%)$; ${ }^{\mathrm{d}}$ Positive in 12 strains $(52 \%)$. 
Table 3. Identification of the 54 rapidly growing mycobacteria strains by phenotypic tests and by INNO-LiPA Mycobacteria v2 assay.

\begin{tabular}{|c|c|c|c|}
\hline Strains & Species $^{\mathbf{a}}$ & Phenotypic tests & INNO-LiPA Mycobacteria v2 \\
\hline M01 & M. fortuitum & M. fortuitum & M. fortuitum - M. peregrinum complex \\
\hline M02 & M. chelonae & M. chelonae & M. chelonae complex (III) \\
\hline M03 & M. fortuitum & M. fortuitum & M. fortuitum - M. peregrinum complex \\
\hline M04 & M. fortuitum & M. fortuitum & M. fortuitum - M. peregrinum complex \\
\hline M05 & M. fortuitum & M. fortuitum & M. fortuitum - M. peregrinum complex \\
\hline M06 & M. fortuitum & M. fortuitum & M. fortuitum - M. peregrinum complex \\
\hline M07 & M. senegalense & M. mageritense $e^{\mathrm{b}}$ & - \\
\hline M08 & M. mисовеnicum & M. mисовеnicum & - \\
\hline M09 & M. abscessus & M. abscessus & M. chelonae complex (III) \\
\hline M10 & M. peregrinum & M. peregrinum & M. fortuitum - M. peregrinum complex \\
\hline M11 & M. alvei & M. fortuitum ${ }^{\mathrm{b}}$ & M. fortuitum - M. peregrinum complex \\
\hline M12 & M. fortuitum & M. fortuitum & M. fortuitum - M. peregrinum complex \\
\hline M13 & M. fortuitum & M. fortuitum & M. fortuitum - M. peregrinum complex \\
\hline M14 & M. chelonae & M. chelonae & M. fortuitum - M. peregrinum complex \\
\hline M15 & M. brumae & M. brumae & - \\
\hline M16 & M. fortuitum & M. fortuitum & M. fortuitum - M. peregrinum complex \\
\hline M17 & M. chelonae & M. chelonae & M. chelonae complex (I, II, III, IV) \\
\hline M18 & M. septicum & M. septicum & M. fortuitum - M. peregrinum complex \\
\hline M19 & M. mageritense & M. mageritense & M. chelonae complex (I, II, III, IV) \\
\hline M20 & M. abscessus & M. abscessus & M. chelonae complex (III) \\
\hline M21 & M. senegalense & M. senegalense & M. fortuitum - M. peregrinum complex \\
\hline M22 & M. fortuitum & M. fortuitum & M. fortuitum - M. peregrinum complex \\
\hline M23 & M. chelonae & M. chelonae & M. chelonae complex (I, II, III, IV) \\
\hline M24 & M. chelonae & M. chelonae & M. chelonae complex (I, II, III, IV) \\
\hline M25 & M. chelonae & M. chelonae & M. chelonae complex (I, II, III, IV) \\
\hline M26 & M. chelonae & M. chelonae & M. chelonae complex (I, II, III, IV) \\
\hline M27 & M. chelonae & M. chelonae & M. chelonae complex (I, II, III, IV) \\
\hline M28 & M. chelonae & M. chelonae & M. chelonae complex (III) \\
\hline M29 & M. chelonae & M. chelonae & M. chelonae complex (I, II, III, IV) \\
\hline M30 & M. smegmatis & M. smegmatis & M. smegmatis \\
\hline M31 & M. fortuitum & M. fortuitum & M. fortuitum - M. peregrinum complex \\
\hline M32 & M. abscessus & M. abscessus & M. chelonae complex (I, II, III, IV) \\
\hline M33 & M. fortuitum & M. fortuitum & M. fortuitum - M. peregrinum complex \\
\hline M34 & M. fortuitum & M. fortuitum & M. fortuitum - M. peregrinum complex \\
\hline M35 & M. fortuitum & M. fortuitum & M. fortuitum - M. peregrinum complex \\
\hline M36 & M. neoaurum & M. neoaurum & 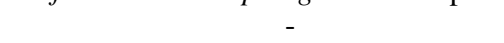 \\
\hline M37 & M. abscessus & M. abscessus & M. chelonae complex (I, II, III, IV) \\
\hline M38 & M. abscessus & M. abscessus & - \\
\hline M39 & M. abscessus & M. abscessus & M. chelonae complex (I, II, III, IV) \\
\hline M40 & M. fortuitum & M. fortuitum & M. fortuitum - M. peregrinum complex \\
\hline M41 & M. fortuitum & M. fortuitum & M. fortuitum - M. peregrinum complex \\
\hline M42 & M. fortuitum & M. fortuitum & M. fortuitum - M. peregrinum complex \\
\hline M43 & M. fortuitum & M. fortuitum & M. fortuitum - M. peregrinum complex \\
\hline M44 & M. fortuitum & M. fortuitum & M. fortuitum - M. peregrinum complex \\
\hline M45 & M. fortuitum & M. fortuitum & M. fortuitum - M. peregrinum complex \\
\hline M46 & M. fortuitum & M. fortuitum & M. fortuitum - M. peregrinum complex \\
\hline M47 & M. chelonae & M. chelonae & M. chelonae complex (I, II, III, IV) \\
\hline M48 & M. fortuitum & M. fortuitum & M. fortuitum - M. peregrinum complex \\
\hline M49 & M. fortuitum & M. fortuitum & M. fortuitum - M. peregrinum complex \\
\hline M50 & M. abscessus & M. abscessus & M. chelonae complex (I, II, III, IV) \\
\hline M51 & M. abscessus & M. abscessus & M. chelonae complex (III) \\
\hline M52 & M. abscessus & M. abscessus & M. chelonae complex (I, II, III, IV) \\
\hline M53 & M. abscessus & M. abscessus & M. chelonae complex (III) \\
\hline M54 & M. fortuitum & M. fortuitum & M. fortuitum - M. peregrinum complex \\
\hline
\end{tabular}

a Identification by PCR of the $16 \mathrm{~S}$ rRNA and $h s p 65$ genes

${ }^{\mathrm{b}}$ Misidentified strains 


\section{DISCUSSION}

Rapidly growing mycobacteria are preferably found in the environment, and rarely in clinic. From our experience, these mycobacteria are more frequently isolated from respiratory secretions and cutaneous abscesses $(18,19)$. The predominant species of RGM in clinic are Mycobacterium fortuitum, $M$. chelonae and M. abscessus; the rest are occasionally found. The most of species belong to the nonchromogen group and to the $M$. fortuitum complex, including $M$. fortuitum, $M$. peregrinum, M. mucogenicum, $M$. senegalense, M. alvei, $M$. houstonense, M. boenickei, M. conceptionense, M. porcinum, M. neworleansense and M. brisbanense (8). In our series we identified 12 different species, seven out of them belonged to this group. We only found a scotochromogen strain of $M$. neoaurum species and none photochromogen.

The conventional mycobacteria identification according to their phenotypical characteristics and biochemical behaviour becomes complex but accessible to any laboratory and of a great utility for RGM differentiation; however, it is not valid for some species. For this reason, the use of phenotypic, biochemical, chromatographic and molecular techniques all together is recommended for their correct identification. Sequencing of the ribosomal RNA $16 \mathrm{~S}$ gene and the study of the polymorphism of the restriction patterns for the identification of RGM show an excellent result, although they are labor-intensive and limited to reference laboratories as they require sophisticated equipment. Alternative methods as INNO-LiPA Mycobacteria v2 assay permit simultaneous identification of 16 species of mycobacteria, including RGM of M. fortuitum-peregrinum complex, M. chelonae and $M$. smegmatis, and are reliable for their use in clinical laboratories $(4,5,7,9,14,15)$.

In accordance with our results, INNO-LiPA Mycobacteria v2 assay presents a sensitivity sensibility of $87.0 \%$ in the identification of RGM. In our series there were two strains uncorrectly identified (M. chelonae and M. mageritense), three strains did not hybridize (M. senegalense, M. mucogenicum and M. abscessus) and other two could not be identified for not being included in the assay (M. brumae and M. neoaurum). We proved that the technique is well suited with mycobacteria within the $M$. fortuitum complex, but it does not differentiate the species inside the group (15); in other respects, the assay was poor discriminative with $M$. abscessus strains. Test results are better and sensitivity close to $100 \%$ in the identification of M. tuberculosis complex and other slow growing mycobacteria $(7,9,14)$.

In conclusion, we find INNO-LiPA Mycobacteria v2 assay of interest for the identification of the most frequent RGM in clinic, it is simple to perform but provides few advantages in comparison with conventional methods and sometimes needs complementary tests to identify Mycobacterium fortuitum complex, M. chelonae complex and specific species. Although the ITS has been shown to be more discriminative than the $16 \mathrm{~S}$ rRNA itself, the heterogeneity in the RGM group requires either ITS or hsp65 gene sequencing for a more precise identification of species.

\section{REFERENCES}

1. Brunello, F.; Ligozzi, M.; Cristelli, E.; Bonora, S.; Tortoli, E.; Fontana, R. (2001). Identification of 54 mycobacterial species by PCR-restriction fragment length polymorphism analysis of the hsp65 gene. J. Clin. Microbiol. 39, 2799-2806.

2. Cramer, J.P.; Sudeck, H.; Burchard, G.D. (2007). Pulmonary infection with rapidly growing mycobacteria in a singer with achalasia: a case report. J. Infect. 54, 219-221.

3. De Groote, M.A.; Uit, G. (2006). Infections due to rapidly growing mycobacteria. Clin. Infect. Dis. 42, 1756-1763.

4. Lebrun, L.; Weill, F.X.; Lafendi, L.; Houriez, F.; Casanova, F.; Gutierrez, C. et al. (2005). Use of the INNO-LiPA-Mycobacteria assay (version 2) for identification of Mycobacterium avium-Mycobacterium intracellulare-Mycobacterium scrofulaceum complex isolates. J. Clin. Microbiol. 43, 2567-2574.

5. Mäkinen, J.; Sarkola, A.; Marjamäki, M.; Viljanen, M.K.; Soini, H. (2002). Evaluation of GenoType and LiPA Mycobacteria assays for identification of finnish mycobacterial isolates. J. Clin. Microbiol. 40, 3478-3481.

6. Meyers, H.; Brown-Elliot, B.A.; Moore, D.; Curry, J.; Truong, C.; Zhang, Y. et al. (2002). An outbreak of Mycobacterium chelonae infection 
following liposuction. Clin. Infect. Dis. 34, 1500-1507.

7. Padilla, E.; González, V.; Manterola, J.M.; Pérez, A.; Quesada, M.D.; Gordillo, S. et al. (2004). Comparative evaluation of the new version of the INNO-LiPA Mycobacteria and GenoType Mycobacterium assays for identification of Mycobacterium species from MB/BacT liquid cultures artificially inoculated with mycobacterial strains. J. Clin. Microbiol. 42, 3083-3088.

8. Schinsky, M.F.; Morey, R.E.; Steigerwalt, A.G.; Douglas, M.P.; Wilson, R.W.; Floyd, M.M. et al. (2004). Taxonomic variation in the Mycobacterium fortuitum third-biovariant complex: Description of Mycobacterium bonickei sp. nov., Mycobacterium houstonense sp. nov., Mycobacterium neworleansense sp. nov., Mycobacterium concordense sp. nov., Mycobacterium brisbanense sp. nov., and recognition of Mycobacterium porcinum from human clinical isolates. Int. J. Syst. Evol. Microbiol. 54, 1653-1667.

9. Suffys, P.N.; Da Silva Rocha, A.; De Oliveira, M.; Dias Campos, C.E.; Werneck Barreto, A.M.; Portaels, F.; Rigouts, L.; Wouters, G.; Jannes, G.; Van Reybroeck, G.; Mijs, W.; Vanderborght, B. (2001). Rapid identification of mycobacteria to the species level using INNO-LiPA Mycobacteria, a reverse hybridization assay. J. Clin. Microbiol. 39, 44774482 .

10. Tortoli, E. (2003). Impact of genotypic studies on mycobacterial taxonomy: the new mycobacteria of the 1990s. Clin. Microbiol. Rev. 16, 319-354.

11. Kox, L.F.F.; Leeuwen, J.; Knijper, S.; Jansen, H.M.; Kolk, A.H.J. (1995). PCR assay based on DNA coding for 16S rRNA for detection and identification of mycobacteria in clinical samples. J. Clin. Microbiol. 33, 3225-3233.

12. Figueiredo, E.E.S.; Carvalho, R.C.T.; Silvestre, F.G.; Lilenbaum, W.; Fonseca, L.S.; Silva, J.T.; Paschoalin, V.M.F. (2010). Detection of
Mycobacterium bovis DNA in nasal swabs from tuberculous cattle by a multiplex PCR. Braz. J. Microbiol. 41, 386-390.

13. Marchi, A.M.; Juttel, I.D.; Kawacubo, E.M.; Dalmarco, E.M.; Blatt, S.L.; Cordova, C.M.M. (2008). Evaluation of methods for detection and identification of Mycobacterium species in patients suspected of having pulmonary tuberculosis. Braz. J. Microbiol. 39, 613-618.

14. Tortoli, E.; Mariottini, A.; Mazzarelli, G. (2003). Evaluation of INNOLiPA Mycobacteria v2: Improved reverse hybridization multiple DNA probe assay for mycobacterial identification. J. Clin. Microbiol. 41, 4418-4420.

15. Trombert-Paolantoni, S.; Poveda, J.D.; Figarella, P. (2004). Comparison de deux techniques d'hybridation moléculaire dans l'identification de mycobactéries en pratique courante. Pathol. Biol. 52, 462-468.

16. Telenti, A.; Marchesi, F.; Balz, M.; Bally, F.; Botrger, E.C.; Bodmer, T. (1993). Rapid identification of mycobacteria to the species level by polymerase chain reaction and restriction enzyme analysis. J. Clin. Microbiol. 31,175-178.

17. Smet, K.A.L.; Brown, I.N.; Yates, M.; Ivanyi, J. (1995). Ribosomal internal transcribed spacer sequences are identical among Mycobacterium avium-intracellulare complex isolates from AIDS patients, but vary among isolates from elderly pulmonary disease patients. Microbiology. 141, 2739-2747.

18. Uslan, D.Z.; Kowalski, T.J.; Wengenack, N.L.; Virk, A.; Wilson, J.W. (2006). Skin and soft tissue infections due to rapidly growing mycobacteria: comparison of clinical features, treatment, and susceptibility. Arch. Dermatol. 142, 1287-1292.

19. Wallace, R.; Brown, B.; Griffith, D. (1998). Nosocomial outbreak/pseudo outbreaks caused by nontuberculous mycobacteria. Annu. Rev. Microbiol. $52,453-490$. 